\title{
Chlorhexidine Hypersensitivity: A Critical and Updated Review
}

\section{Calogiuri GF ${ }^{1}$, Di Leo $E^{2-4}$, Trautmann $A^{5}$, Nettis $E^{6 *}$, Ferrannini $A^{6}$ and Vacca $A^{3,6}$}

$14^{\text {th }}$ Pneumology Department, Pneumologic Hospital A Galateo San Cesario di Lecce, Lecce, Italy

2Section of Allergology and Clinical Immunology, Unit of Internal Medicine, "F. Miulli" Hospital, Acquaviva delle Fonti, Bari, Italy

${ }^{3}$ Clinical Medicine "G. Baccelli", Department of Internal and Specialist Medicine, University of Bari Medical School, Bari, Italy

${ }^{4}$ Department of Biomedical Sciences and Human Oncology, University of Bari Medical School, Bari, Italy

${ }^{5}$ Allergy Unit, Department of Dermatology, University of Würzburg, Germany

${ }^{6}$ Section of Allergy and Clinical Immunology, Department of Internal and Specialist Medicine, University of Bari Medical School, Bari, Italy

\begin{abstract}
Chlorhexidine is a synthetic bis-biguanide widely used as disinfectant in medical and surgical fields, highly appreciated for its efficacy, microbicide properties and low costs. Unfortunately, Chlorhexidine can be responsible for hypersensitivity reactions (from contact dermatitis to life-threatening anaphylaxis) but its role as allergen, often complicating a perioperative or anesthetic session, is still undervalued and misdiagnosed. In the lights of the most recent studies and case reports published, hereby we have comprehensively reviewed the main aspects of Chlorhexidine hypersensitivity, including, pathway of sensitization, cross-reactivity and new diagnostic laboratory tools
\end{abstract}

Keywords: Chlorhexidine; Contact anaphylaxis; Contact dermatitis; Disinfectant; Hypersensitivity reactions

\section{Introduction}

\section{Chemistry}

Chlorhexidine (1:6-di[4-chlorophenyldiguanido]-hexane) (CHL) is a synthetic topical disinfectant industrially produced since 1954. It is a chlorophenyl-bis-biguanide containing two chloroguanide chains linked by a hexamethylene chain (Figure 1). It is a strong base, and at physiological $\mathrm{pH}$ a dication. It is usually insoluble in water so it needs to be formulated with either gluconic or acetic acid to form watersoluble digluconate or diacetate salts. CHL solutions are colourless and odourless, but have an extremely bitter taste.

\section{Pharmacology features}

If topically used, CHL covalently binds to cutaneous and mucosal proteins resulting in a persisting antimicrobial effect with limited systemic absorption, even after its oral ingestion [1]. CHL has bacteriostatic, bactericidal and fungicidal activity towards a wide range of micro-organisms. It is adsorbed on phosphate-containing protein components of the bacterial cell wall and by penetrating and breaking the bacterial cytoplasmic membrane CHL provokes the leakage of cytoplasmic components. The higher is its concentrations, the more

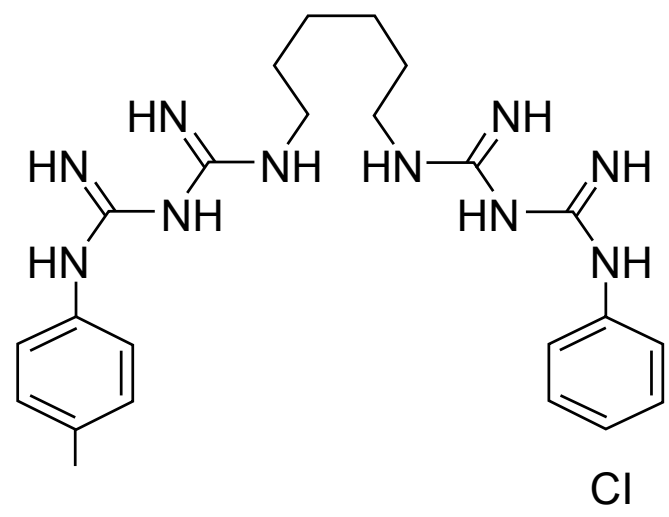

Figure 1: Chlohexidine. bactericidal effect it exerts on bacteria [1]. For this reason, CHL concentration in aqueous or alcoholic pharmaceutical solutions ranges from $0.004 \%$ to $4 \%$. Prolonged exposure increases the bactericidal effect against most bacteria. CHL activity is regularly reduced by the presence of organic compounds, such as fatty acids, and at lower $\mathrm{pH}$ [2].

\section{Medical application}

CHL, especially as digluconate ester, is widely used in various topical applications (mouthwash solutions, dental gels and toothpaste) for its capability to bind oral mucosal surfaces inhibiting dental plaque formation [1]. Importantly, CHL is deactivated by anionic compounds, including the anionic surfactants commonly present as detergents in toothpastes and mouthwashes. Therefore, CHL mouth rinsing solutions should be used at least 30 minutes after other dental products. CHL can be found yet in plasters and dressings, ointments, and suppositories, contraceptive gels and it is available as an over-the-counter solution for disinfection of minor cuts and wounds [1]. CHL acts as preservative agent in various liquid soaps, shower foams, cosmetics, toothpaste, lubricants and medical ointments since it prevents bacterial contamination [1]. Because CHL significantly decreases bacterial skin colonization it also finds a broad use in surgical fields as topical disinfectant applied onto prior to surgical incision.

CHL-alcohol combination as an antiseptic solution has showed to be more effective and superior than iodine for hand-washing and surgical skin preparation [3]. The use of CHL as a skin disinfectant reduces the incidence of intra-vascular catheter-related bloodstream infections [1].

*Corresponding author: Eustachio Nettis, Section of Allergy and Clinical Immunology, University of Bari Medical School, Bari, Policlinico, Piazza Giulio Cesare, 11, 70124 Bari, Italy, Tel: +39 080 5592821; Fax: +39 0805593576 ; E-mail: e.nettis@allergy.uniba.it

Received February 15, 2013; Accepted June 25, 2013; Published July 01, 2013

Citation: Calogiuri GF, Di Leo E, Trautmann A, Nettis E, Ferrannini A, et al. (2013) Chlorhexidine Hypersensitivity: A Critical and Updated Review. J Allergy Ther 4: 141. doi:10.4172/2155-6121.1000141

Copyright: @ 2013 Calogiuri GF, et al. This is an open-access article distributed under the terms of the Creative Commons Attribution License, which permits unrestricted use, distribution, and reproduction in any medium, provided the original author and source are credited. 
Various medical tools such as urinary or central venous catheters and cannula are CHL-coated to optimize their sterilization. In aseptic environments even medical dressings could be impregnated by CHL solutions [1]. CHL can be also used for bladder or intrauterine irrigation, before inserting contraceptive devices for instance.

Diacetate CHL can be found as a preservative in products such as antacid preparations, contact lens fluids and cosmetics. It has also been used in commercial food handling [1] and it is present in household antiseptic products.

\section{Route of application}

CHL is usually prepared for topical skin and mucosal use only excluding systemic administration. After accidental intravenous administration, $\mathrm{CHL}$ caused a toxic acute respiratory distress syndrome [4] also at a low dosage. Topically applied CHL is usually well tolerated. Nevertheless, properly applied CHL seems to be responsible for several serious life-threatening immediate-type hypersensitivity reactions.

\section{Chlorexidine Hypersensitivity: Clinical Aspects}

\section{Contact dermatitis}

CHL can induce allergic contact dermatitis at the site of application. The first case of well described CHL contact dermatitis has been reported in 1972 [5]. The allergic nature of the dermatitis was confirmed by epicutaneous and intradermal testing with various CHL salts and excipients at different concentrations. The identification of $\mathrm{CHL}$ as a contact allergen provoked further studies recruiting more cases, especially patients with ulcers and stasis eczema [6,7]; CHL contact sensitization generally appears after prolonged and repeated applications [7]. For physical-chemical reasons false negative patch tests have been obtained when CHL was applied in petrolatum $[8,9]$. Since CHL is mainly active in aqueous solution, several Authors have investigated which CHL salt is suitable for patch testing and tried to determine the optimal patch test concentration [10]. One study was performed on 297 patients, most of them suffering from leg ulcers. Thirty-nine patients with CHL contact sensitization were identified. The acetate CHL $1 \%$ in aqueous solution produced more reactions than gluconate $1 \%$, but many test reactions appeared to be irritant. Finally, the Authors suggested acetate CHL $0.05 \%$ in aqueous solution as a suitable test reagent for the screening purposes [10]. Furthermore other components contained in a CHL solution may be responsible for contact allergy. In fact, allergic contact dermatitis to phenolsulfonphthalein [11] and contact eczema due to a curcumin dye obtained by Curcuma longa roots [12] both components of the CHL solutions have been reported. The curcumin dye has been added to provide a yellow color to some CHL solutions used to skin disinfection prior to surgery [12] to avoid accidental ingestion [13]. Since CHL is a colorless solution a dye facilitates visually the cutaneous disinfection procedure. Curcumin $1 \%$ in petrolatum or $0.05 \%$ in ethanol gave positive patch test reactions [12]. There are only single case reports of connubial dermatitis [14], photodermatitis [15] and fixed drug eruption [16] induced by CHL.

\section{Immediate type hypersensitivity}

CHL may induce immediate-type hypersensitivity reactions either by topical application on mucosa or skin or by the insertion of urethral or central venous CHL-coated catheters. CHL allergy may complicate and cause the interruption of surgical procedures and anaesthesia sessions. Because CHL is an underestimated allergen several anaphylactic episodes may occur in a patient before the identification of CHL as the responsible allergen [17-19].

\section{Topical agents and contact anaphylaxis}

Topical chlorhexidine may causes anaphylaxis, especially when applied on mucosal surfaces. Among 50 cases of adverse reactions to CHL due to mucosal application, 9 cases of anaphylactic shock were reported by the Japanese Ministry of Welfare between 1967 and 1984 [20]. Even application of CHL on small mucosal areas could be sufficient for triggering an IgE mediated anaphylaxis as in a reported case of anaphylaxis following topical cleaning of nasal mucosa with a gluconate CHL $0.05 \%$ solution in a 53 year old man admitted for a trans-sphenoidal resection of a pituitary adenoma [21]. Vaginal instillation of CHL solution or CHL containing-gel application while placing an intrauterine device or during cervix conisation can result in an anaphylactic shock too [10,22]. Generalized urticaria following urethral instillation of CHL has been referred too, potentially evolving towards more serious life-threatening anaphylactic symptoms if not stopped by adequate emergency therapy. Authors suggested that such reactions are underreported and alternative not cross-reacting antiseptics are requested for urological and gynecological procedures [23]. Surprisingly, simple contact urticaria which can be considered as an initial sign of IgE-mediated contact anaphylaxis induced by CHL has been rarely reported $[8,9]$.

When applied on burn injured skin [24], on minor excoriations [25] or even small open wounds [26] there is an increased risk for CHLtriggered anaphylaxis. Subclinical thinness of the stratum corneum by repeated frictions may be sufficient to increase CHL adsorption on an apparently intact skin as it happened to a $33 \mathrm{y}$-old man after a horseride walk [27].

Anaphylactic CHL reactions have been also reported in patients with healthy skin $[28,29]$. In studies using radioisotopes CHL was shown to penetrate easily the mucosal surface [30], whereas $2 \% \mathrm{CHL}$ aqueous solution seems to permeate poorly into deeper layers of skin two minutes after application [31]. However, alcoholic solutions may cause dehydration of stratum corneum proteins, thus potentially worsening the CHL permeation into the dermal skin compartment [32]. Several other contributing factors such as the site of application, the concentration of CHL solution [33] or the body surface area involved during pre-operative disinfection should be considered, as suggested by the report of a patient who experienced contact anaphylaxis with respiratory arrest following a whole body bath with gluconate $\mathrm{CHL}$ $0.05 \%[34]$.

Peri-operative anaphylaxis symptoms generally appear immediately within the first 15-45 minutes after the beginning of anaesthesia. The initial symptoms are often underestimated as simple acute urticaria or not recognized due to surgical coverage of the body. But generalized urticaria may develop rapidly to systemic anaphylaxis with symptoms including tachycardia, bronchospam, and hypotension. Without proper and fast treatment the cascade evolves to severe anaphylactic shock due to cardiovascular collapse and finally cardiac or respiratory arrest [33]. Kounis syndrome, a myocardial ischemia induced by a vasospasm associated to anaphylaxis, has been described after application of digluconate $\mathrm{CHL} 2 \%$ in a patient undergoing resection of the upper lobe of the left lung because of adenocarcinoma [35] and in a 43 year old non atopic man after disinfection of a drain insertion site [36].

Sometimes delayed-type reactions such as allergic contact dermatitis and immediate-type reactions may coexist in the same patient $[17,36-$ 38]. CHL-induced eczema may precede the development of CHLinduced anaphylaxis by years, suggesting that patients with CHLinduced contact dermatitis are prone to IgE sensitization. Therefore 
in patients with allergic CHL-contact delayed-type hypersensitivity further use of CHL or CHL-coated catheters should be avoided to prevent IgE sensitization [36].

Even simple but invasive procedures as digital rectal examination with CHL $0.05 \%$ can result in an anaphylactic reaction, firstly attributed to natural rubber latex hypersensitivity [39], although contemporary latex and CHL sensitization in the same patient has been exceptionally described [22].

Rare reports are published about cutaneuos adverse reactions following the use of CHL in mouth-wash rinses such as fixed drug eruption [16] or contact stomatitis [40]. However, urticaria [41] and anaphylaxis [17] have been reported after the use of CHL mouth-wash rinses too. The oral route may be a potential and undervalued pathway of sensitisation to $\mathrm{CHL}$, especially with the prolonged use of topical CHL-containing antiseptic solution [42].

\section{Catheter devices and peri-operative anaphylaxis}

The first report of documented CHL allergy due to a urethral gel dates back to 1992 [43]. Thereafter, some cases of rapidly evolving anaphylactic reactions induced by use of CHL gel coated urethral catheters have been described in male patients [18,44-50]. As far as the use of CHL impregnating central venous catheters (CVC) is concerned, an immediate-type adverse reaction has been firstly described in 1997 in a 47 year old Japanese woman, suggesting CHL released by CVC may be sufficient to provoke symptoms [51]. CHL impregnated CVCs are widely used, because CHL gluconate $2 \%$ has demonstrated to reduce significantly intravascular catheter-related infections [52], but CHL-coated CVC may be an important unrecognized source of CHL exposure.

In most of the case reports, patients experienced at least two episodes of peri-operative anaphylaxis despite CHL had been correctly identified as the responsible allergen and avoided in disinfectants and urethral gels during the second anaesthesia session [19,53-55]. It is possible also that CHL hypersensitivity, carefully reported by the patient, has been undervalued by anesthesiologists during the placement of a CVC via the femoral vein [56].

Moreover, contemporary double exposure to CHL-coated catheters may be possible: through central venous line plus the urethral pathway [57], so inducing an immediate-type adverse reaction which complicated the surgical procedure of a dissecting thoracic aortic aneurism in a 74 years old man, already haemodinamically unstable [57].

Recently, studies involving cohorts of patients with CHL-induced anaphylactic reactions following the placement of urethral catheters $[47,50]$ or CVC [58], have been published, suggesting either an increased attention to the problem from anaesthesiologists or an augmented use of CHL in medical devices. Furthermore, in most of patients with CHLinduced anaphylaxis, some previous mild reactions following $\mathrm{CHL}$ exposure could be retrospectively identified in their clinical history. These symptoms were undervalued or misdiagnosed, being attributed to a vaso-vagal reaction or to a non-allergic erythematous urticarial rash due to drugs with a histamine-releasing effect $[17,18,28,36,48,50]$.

During anaesthesia every procedure and drug administration should be recorded and annotated step by step in the patient's clinical diary: that may help to identify the causative agent in case of perioperative anaphylaxis [59]. Importantly, CHL is not documented as a drug administered by anaesthesiologists because skin disinfection and catheter insertion performed by nurse staffs are considered as routinely preoperative activities.

\section{Pathomechanism of Immediate-Type Hypersensitivity}

\section{IgE-mediated CHL hypersensitivity}

In 1984, Nishioka et al. [60] firstly suspected an IgE-mediated pathomechanism in CHL hypersensitivity. They described a boy with intraoperative anaphylactic shock after topical disinfection with CHL. Positive Prausnitz-Küstner test, positive SPT response using CHL $0.05 \%$, and positive histamine release test confirmed indirectly an IgEmediated mechanism [60]. Two years later, Ohtashi et al. elaborated a Radio-Allergo-Sorben-Test (RAST) method to detect CHL-specific IgE antibodies in vitro from the sera of eight individuals with a previous CHL-induced anaphylaxis. In these patients the symptoms of CHL anaphylaxis were attributed to cutaneous, mucosal (including respiratory) and systemic exposure [29]. CHL has a molecular weight of $505 \mathrm{Da}$ and usually interacts only electrostatically with proteins.

Layton et al. proposed that $\mathrm{N}$-chlorobiguanide derivatives covalently conjugate with tyrosine, lysine and tryptophan residues, probably via nucleophilic groups [61]. This knowledge allowed the production of a better defined sem- CHL-human serum albumin (HSA) conjugate which allowed detecting CHL-specific IgE more efficiently in sera of Japanese patients who had experienced anaphylactic reactions [61]. Interestingly, such a conjugate allowed the identification IgG to CHL even in professionally exposed English health care personnel [61].

Pham et al. tried to identify immunogenic epitopes of CHL molecule after isolation of CHL specific IgE, either by RAST method on sepharose or by its conjugates [62]. The serum belonged to a patient who had experienced three life-threatening episodes of anaphylaxis during anesthesia before the culprit agent was correctly identified [62].

The RAST inhibition study revealed the lack of IgE affinity towards compounds which mimiced the terminal 4-chlorophenol group of CHL, while compounds like chlorguanide or proguanil, an antimalarial medication, which is half the CHL molecule (Figure 2) and alexidine (Figure 3 ) showed a significant inhibition of IgE binding to CHL-sepharose (34\% and $40 \%$ respectively) [62]. The unmodified CHL molecule showed the highest IgE affinity (81\% inhibition of IgE<smiles>CC(C)NC(=N)NC(=N)Nc1ccc(Cl)cc1</smiles>

Figure 2: Proguanil.

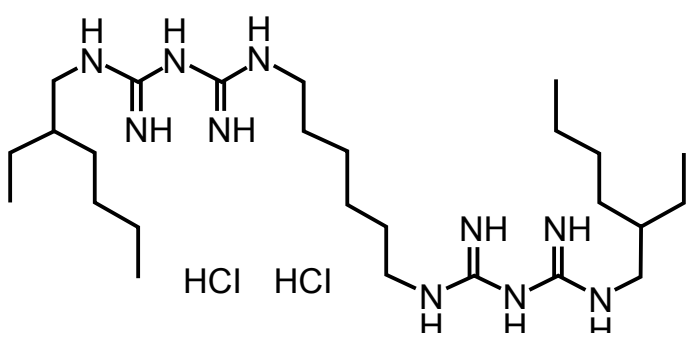

Figure 3: Alexidine. 


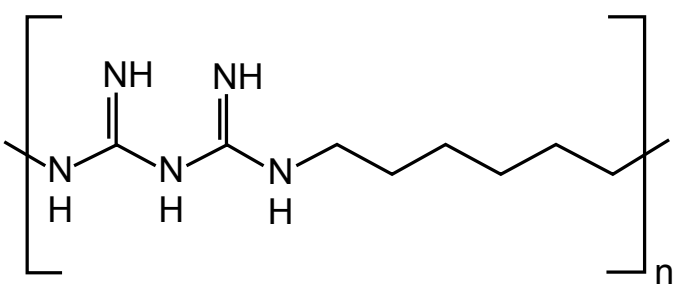

Figure 4: Plyhexanide.

binding to CHL-sepharose). Therefore, Authors concluded despite the whole CHL molecule should have been considered as allergenic, the structure complementarity determining region of $\operatorname{IgE}$ is directed to the hexamethylene biguanide present in both CHL and alexidine [62].

Previous studies, using CHL specific murine IgG antibodies, had found that $\mathrm{N}$-chlorination of CHL did not affect its allergenicity and CHL could be considered as a bivalent hapten like succinylcholine [63]. In the lights of these findings, CHL, bridging between two bound IgE antibodies, is able to efficiently trigger cutaneous mast-cells with the release of vasoactive mediators.

The importance of the hexamethylene group as major allergenic determinant was confirmed by reports regarding anaphylactic reactions induced by polyhexanide $[64,65]$, which is widely used in surgical field as topical disinfectant. Polyhexanide is a CHL derived polymer, whose chemical structure is very similar to CHL (Figure 4). Nevertheless, in both the two patients who had developed a severe anaphylactic reaction following contact of surgical wounds with polyhexanide, skin prick test resulted positive to polyhexanide, but not to CHL [64], although from their clinical history, CHL was the original sensitizing agent [64].

Recently Kautz et al. published a case of a 81 year old female patient with a history of anaphylaxis following the use of a new brand of toilet paper containing polyhexanide [66].

Skin prick test gave positive responses with polyhexanide and $\mathrm{CHL}$ and specific serum IgE to both disinfectants were isolated from the patient, but RAST inhibition indicated only limited in vitro crossreactivity between the two molecules [66].

Authors supposed that patients with known CHL hypersensitivity may be at risk for allergic reactions to polyhexadine, but surprisingly, in sera from three patients with a history of CHL allergy, no specific polyhexanide-IgE antibodies were detected [66].

ELISA inhibition data with murine anti-CHL IgG antibodies had already indicated the relative importance of the $\mathrm{p}$-chlorophenyl epitope compared to the biguanide hexamethylene structure. This may be a consequence of the spatial conformation of the CHL hapten on both the immunogen and ELISA antigen [63]. In this respect, N-chlorination of $\mathrm{CHL}$ resulted in the formation of $\mathrm{N}$-chloro biguanide derivatives which can bind covalently to certain nucleophilic functional groups of proteins [62]. N-Chlorine group may partially obscure most of the biguanide hexamethylene structures or it makes them sterically inaccessible [63].

Probably these results explain why alexidine demonstrated an $\operatorname{IgE}$ affinity higher (40\%) than chlorguanide, i.e. half CHL molecule (34\%) [62] and why IgE to polyhexanide have a lower affinity to CHL [66].

\section{Risk factors for sensitization}

Professional exposure may represent an important source of CHL sensitisation among health care workers, as suggested since 1989 by the onset of an occupational asthma to CHL in two nurses, whose diagnosis was confirmed by bronchial provocation test, although no skin tests were carried out in these patients [67].

The risk of sensitization and allergy to CHL in health care workers is not well established yet. A Japanese study performed on 307 healthcare workers found 89 of them describing an occupational allergy such as contact dermatitis, allergic rhinitis, bronchial asthma and overlap symptoms [68]. Contact dermatitis was the most prevalent clinical manifestation, while CHL proved to be the second prevalent agent inducing occupational allergy, after rubber gloves [68]. In contrast, a Danish study investigated the prevalence of IV-type and I-type hypersensitivity reactions to CHL in a group of 104 health care workers by performing skin patch test, skin prick test and intradermal test [69]. They failed to demonstrate any evidence of I-type or IV-type sensitization to CHL [69], although a previous investigation of the same Authors had identified CHL as the most common cause of anaphylaxis during anesthesia sessions, with a prevalence exceeding 13\% [70].

Recently, a study performed by distributing a specific questionnaire to 86 health care operators at Queen Elizabeth Hospital Woolwich in London, detected 4 cases of IgE-mediated CHL allergy among the 53 collaborating operators [71]. Allergy was confirmed by measurement of CHL serum specific IgE and positive skin prick tests using gluconate CHL $0.5 \%$ and $1 \%$ in aqueous solution [71].

Authors speculated that a higher incidence of CHL hypersensitivity in English health care workers than that the described in Danish professionally exposed subjects could be attributed to the different habits in hands disinfection. In fact, Danish health workers used a 0.5-1.0\% CHL hand wash solution, but English health operators were exposed to a $4 \%$ CHL hand wash disinfectant. This hypothesis seems to be supported by the shortly onset of allergic symptoms such as contact urticaria or erythema, involving mainly hands and forearms after handling CHL solution [71].

Furthermore it has been evidenced that even the use of CHLcontaining cosmetics and topical drug (i.e. corticosteroid ointments) may promote CHL contact allergy [72].

Because diabetic patients show a higher incidence of oral infections, either bacterial or fungal, they are compelled to use CHL mouth rinses more frequently than other patients. For this reason, topical adverse events due to the prolonged use of oral CHL applications have been often reported in these patients [73]. Diabetics assume biguanides, i.e. phenformin and metformin, as oral drugs [74], whose chemical formula is very similar to CHL (Figures 5 and 6).

A recent investigation on the different incidence of allergy to neuromuscular blocking agents (NMBA) used in anesthesia between Norway and Sweden identified in pholcodine, a common cough syrup sold like an over-the-counter product in Norway but not in Sweden, the responsible agent of hidden sensitization to NMBAs [75]. Authors showed that frequent consumption of pholcodine

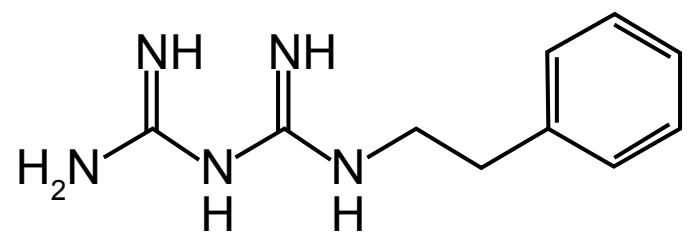

Figure 5: Phenformin 


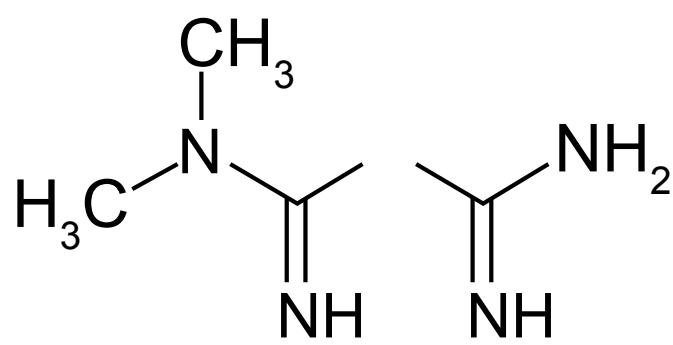

Figure 6: Metformin.

containing cough mixture was related to a high presence of $\operatorname{IgE}$ sensitization to pholcodine, morphine and, partially, suxamethonium, all pharmacological molecules sharing quaternary ammonium groups which are the immmunogenic determinants of NMBAs [75].

The specific IgE to pholcodine increased after 7 days therapy with the cough syrup containing pholcodine, by demonstrating oral pathway was another neglected sensitization route to NMBAs in Norwegians [75]. In the light of pholcodine's experience, also anti-diabetic drugs could be potentially sensitizing agents and consequently diabetic patients should be considered at risk for CHL allergy even. Diabetes has been described and reported poorly in patient with CHL allergy, probably because it is not considered important in clinical history of perioperative anaphylaxis, as atopy or previous drug allergy [19,39]

At last, ethnicity may play a role and Japanese and Asian peoples seem to be more susceptible to develop an IgE-mediated immune response $[9,20,29,39,42,47,51,53,60,61,68]$ to $\mathrm{CHL}$ than Caucasians, but this tendency could be due to different levels of exposure even.

\section{Allergic Investigations}

Garvey et al. [69] identified 12 subjects out of 174 patients who had experienced perioperative anaphylaxis from 1999 to 2005 with positive skin prick tests with gluconate $\mathrm{CHL} 0.5 \%$ and positive intradermal test using gluconate CHL $0.0002 \%$. In 11 of these twelve patients CHL specific serum IgE were detected by ImmunoCap method (Phadia AB Inc. Uppsala - Sweden) prepared by covalent coupling 1-[-N5-(pchlorophenyl)biguanido]-6-aminohexane, i.e., half the CHL molecule) to a cyanogen bromide-activated sponge. They hypothesized that CHL specific IgE antibodies tend to decrease far from the adverse reaction, so they should be dosed within six months [76]. Such a possibility has already been suggested previously [29]. The relationship between specific time of IgE measurement and exposure is important to detect properly CHL-specific IgE. It has been demonstrated that clinically relevant sensitization could still be present at a low IgE specific titres corresponding to $0.20-0.35 \mathrm{kUA} / \mathrm{L}$, especially if $\mathrm{CHL}$ exposure has been avoided for a long period [71]. This value is below the defined normal cut-off commercially available specific IgE kits. Probably, for that reason, Garvey et al failed to detect CHL specific IgE in skin tests and serum samples of 10 other patients in whom CHL was strongly suspected as causative allergen [76]. That study confirmed the validity of the ImmunoCAP assay for the CHL allergy diagnosis since the results of this method is well-related to positive skin tests and tryptase levels [76]. The dosage of mast cell tryptase had showed increased serum values in different reports [17-19,50,54-58,76].

A more recent study performed in England on 6 male patients with a history of anaphylaxis following urinary catheter or a CVC insertion suggested CHL ImmnoCap assay should be reputed an efficient test to detect CHL specific IgE [77], because the ImmunoCap assay revealed
CHL specific IgE levels ranging from $2,3 \mathrm{kUA} / \mathrm{lt}$ up to $30 \mathrm{kUA} /$ lt in patients. In that way, serum IgE values showed great individual variation, not related to severity of reaction or to initial IgE level, as already suggested by Garvey et al. [76].

Previously the validity of ImmunoCAP method has been compared to skin tests and Basophil Activation Test (BAT) [77,78], but serum specific IgE dosage by ImmunoCap resulted more efficient and technically easier to perform [78] than other laboratory tests as sulfidoleukotriene stimulation test (CAST), lymphocyte transformation test and BAT, which have been successfully utilized to investigate CHL hypersensitivity in past case reports $[26,36,49]$.

Although different studies and case report observed a decline of CHL specific IgE levels over time with varying rate [29,76,79], in patients who have experienced an anaphylactic shock, a boosted response with an increase of specific and total $\operatorname{IgE}$ has been found two-three days after the acute adverse event. That phenomenon was documented for beta-lactams [80,81] and ethylene oxide [82], but it could be worth for chlorhexidine allergy too, thus allowing the quick identification of responsible allergen when skin tests cannot be carried out because patient is poorly responsive to skin tests [51]. It should allow reducing the period of allergic follow-up and investigation in cancer patients, for instance.

In another study performed in Finland from 1995 to mid-2001, 1314 patients were skin prick tested with CHL digluconate and their clinical history was investigated deeply, looking for a previous exposure to CHL. 470 patients till 1998 were tested with CHL 1\% in aqueous solution, while the remaining 844 at $0.5 \%$ dilution [83]. Authors found 33 patients (16 females, 17 males; age 1-69 years old) with a positive skin prick test to gluconate CHL [83]. Only 20 subjects showed clinical signs of hypersensitivity, while 13 were asymptomatic. In their clinical history, 16 of them used CHL to treat acne or dermatitis, 3 patients underwent gynecologic examinations or colonscopy, 6 had been subjected to some surgical procedure (heart catheterisation, urologic or orthopaedic operations) [83]. In 8 patients it was not possible to identify the source of CHL exposure, while 2 patients had previously performed orthodontic treatments and 2 patients used CHL solutions for hands disinfection [83]. Furthermore the study seemed to confirm observations of Danish Authors about the possibility that CHL allergy may be more prevalent in patients undergoing surgery or invasive procedures [17], thus resembling latex allergy, which is more frequent in children affected by spina bifida or urogenital malformations because of the multiple corrective operations [84]

In their medical history a lot of patients have reported a recent invasive diagnostic procedure or a surgical operation, including periodontal treatments, in the past two years [17,19,39,47,50,56$58,77,83]$, suggesting that such an aspect should be more carefully investigated in the case history.

Furthermore, there are few patients who are exposed to unknown hidden sources of CHL (through an unreported professional exposure or through cosmetics and mouthwashes abuse?) and they maintain detectable IgE level in serum, not showing the gradual IgE decline seen in most of patients $[76,83]$. Finally, gender influences the sensitization pathway: male patients are mainly sensitized through the urethral catheterisation [17,43-50,76,79], while female patients are sensitized more frequently through a professional exposure $[67,71,85]$.

\section{Concluding Remarks}

CHL hypersensitivity seems to be very frequent and an increasing 
attention is dedicated to this disinfectant as potential allergen $[71,77,85]$ complicating general anesthesia, despite the real incidence of immediate-type adverse reactions is still unknown and underestimated.

When allergic investigations for muscle relaxants and natural rubber latex after perioperative anaphylaxis remain negative, anesthesiologists' and allergists' attention should be focussed on CHL as a hidden allergen [47], because diagnostic tools as skin tests and serum specific IgE assay to identify CHL hypersensitivity are available, but firstly, it needs to suspect correctly the allergen involvement. Although recent anaesthesia guidelines suggest to let skin disinfectant be completely dry before beginning an invasive procedure [86], the cutaneous adsorption or the possibility to introduce CHL with CHL-coated catheters through mucosal or intravenous route neutralises that precaution. More studies are needed to establish the predictive value of skin tests in patients reporting potential risk factors for CHL hypersensitivity as: i) a CHLinduced contact dermatitis; ii) a professional exposure to disinfectants; iii) previous invasive medical procedures in patient's clinical history.

\section{References}

1. Lim KS, Kam PC (2008) Chlorhexidine--pharmacology and clinical applications Anaesth Intensive Care 36: 502-512.

2. McDonnell G, Russell AD (1999) Antiseptics and disinfectants: activity, action, and resistance. Clin Microbiol Rev 12: 147-179.

3. Darouiche RO, Wall MJ Jr, Itani KM, Otterson MF, Webb AL, et al. (2010) Chlorhexidine-Alcohol versus Povidone-lodine for Surgical-Site Antisepsis. N Engl J Med 362: 18-26.

4. Ishigami S, Hase S, Nakashima H, Yamada H, Dohgomori H, et al. (2001) Intravenous chlorhexidine gluconate causing acute respiratory distress syndrome. J Toxicol Clin Toxicol 39: 77-80.

5. Ljunggren B, Möller H (1972) Eczematous contact allergy to chlorhexidine. Acta Derm Venereol 52: 308-310.

6. Osmundsen PE (1982) Contact dermatitis to chlorhexidine. Contact Dermatitis 8: 81-83.

7. Lasthein Andersen B, Brandrup F (1985) Contact dermatitis from chlorhexidine Contact Dermatitis 13: 307-309.

8. Fisher AA (1989) Contact urticaria from chlorhexidine. Cutis 43: 17-18.

9. Wong WK, Goh CL, Chan KW (1990) Contact urticaria from chlorhexidine. Contact Dermatitis 22: 52.

10. Knudsen BB, Avnstorp C (1991) Chlorhexidine gluconate and acetate in patch testing. Contact Dermatitis 24: 45-49.

11. Kaminska R, Mörtenhumer M (2007) Allergic contact dermatitis from phenolsulfonphthalein in chlorhexidine solution. Contact Dermatitis 56: 358360 .

12. Fischer LA, Agner T (2004) Curcumin allergy in relation to yellow chlorhexidine solution used for skin disinfection prior to surgery. Contact Dermatitis 51: 39-40.

13. Mucklow ES (1988) Accidental feeding of a dilute antiseptic solution (chlorhexidine $0.05 \%$ with cetrimide $1 \%$ ) to five babies. Hum Toxicol 7: 567 569

14. Barrazza V (2001) Connubial allergic contact balanitis due to chlorhexidine. Contact Dermatitis 45: 42.

15. Wahlberg JE, Wennersten G (1971) Hypersensitivity and photosensitivity to chlorhexidine. Dermatologica 143: 376-379.

16. Moghadam BK, Drisko CL, Gier RE (1991) Chlorhexidine mouthwash-induced fixed drug eruption. Case report and review of the literature. Oral Surg Oral Med Oral Pathol 71: 431-434.

17. Garvey LH, Roed-Petersen J, Husum B (2001) Anaphylactic reactions in anaesthetised patients - four cases of chlorhexidine allergy. Acta Anaesthesiol Scand 45: 1290-1294.

18. Wicki J, Deluze C, Cirafici L, Desmeules J (1999) Anaphylactic shock induced by intraurethral use of chlorhexidine. Allergy 54: 768-769.
19. Stephens R, Mythen M, Kallis P, Davies DW, Egner W, et al. (2001) Two episodes of life-threatening anaphylaxis in the same patient to a chlorhexidinesulphadiazine-coated central venous catheter. $\mathrm{Br} J$ Anaesth 87: 306-308.

20. Okano M, Nomura M, Hata S, Okada N, Sato K, et al. (1989) Anaphylactic symptoms due to chlorhexidine gluconate. Arch Dermatol 125: 50-52.

21. Chisholm DG, Calder I, Peterson D, Powell M, Moult P (1997) Intranasal chlorhexidine resulting in anaphylactic circulatory arrest. BMJ 315: 785.

22. Porter BJ, Acharya U, Ormerod AD, Herriot R (1998) Latex/chlorhexidineinduced anaphylaxis in pregnancy. Allergy 53: 455-457.

23. Stables GI, Turner WH, Prescott S, Wilkinson SM (1998) Generalized urticaria after skin cleansing and urethral instillation with chlorhexidine-containing products. Br J Urol 82: 756-757.

24. Evans RJ (1992) Acute anaphylaxis due to topical chlorhexidine acetate. BMJ 304: 686.

25. Torricelli R, Wüthrich B (1996) Life-threatening anaphylactic shock due to skin application of chlorhexidine. Clin Exp Allergy 26: 112

26. Krautheim AB, Jermann TH, Bircher AJ (2004) Chlorhexidine anaphylaxis: case report and review of the literature. Contact Dermatitis 50: 113-116.

27. Autegarden JE, Pecquet C, Huet S, Bayrou O, Leynadier F (1999) Anaphylactic shock after application of chlorhexidine to unbroken skin. Contact Dermatitis 40: 215.

28. Cheung J, O'Leary JJ (1985) Allergic reaction to chlorhexidine in an anaesthetised patient. Anaesth Intensive Care 13: 429-430.

29. Ohtoshi T, Yamauchi N, Tadokoro K, Miyachi S, Suzuki S, et al. (1986) IgE antibody-mediated shock reaction caused by topical application of chlorhexidine. Clin Allergy 16: 155-161.

30. Winrow MJ (1973) Metabolic studies with radiolabelled chlorhexidine in animals and man. J Periodontal Res Suppl 12: 45-48.

31. Karpanen TJ, Worthington T, Conway BR, Hilton AC, Elliott TS, et al. (2008) Penetration of chlorhexidine into human skin. Antimicrob Agents Chemother 52: 3633-3636.

32. Van der Merwe D, Riviere JE (2005) Comparative studies on the effects of water, ethanol and water/ethanol mixtures on chemical partitioning into porcine stratum corneum and silastic membrane. Toxicol In Vitro 19: 69-77.

33. Beaudouin E, Kanny G, Morisset M, Renaudin JM, Mertes M, et al. (2004) Immediate hypersensitivity to chlorhexidine: literature review. Eur Ann Allergy Clin Immunol 36: 123-126.

34. Snellman E, Rantanen T (1999) Severe anaphylaxis after a chlorhexidine bath J Am Acad Dermatol 40: 771-772.

35. Conraads VM, Jorens PG, Ebo DG, Claeys MJ, Bosmans JM, et al. (1998) Coronary artery spasm complicating anaphylaxis secondary to skin disinfectant Chest 113: 1417-1419.

36. Ebo DG, Stevens WJ, Bridts CH, Matthieu L (1998) Contact allergic dermatitis and life-threatening anaphylaxis to chlorhexidine. J Allergy Clin Immunol 101 128-129.

37. Bergqvist-Karlsson A (1988) Delayed and immediate-type hypersensitivity to chlorhexidine. Contact Dermatitis 18: 84-88.

38. Lauerma Al (2001) Simultaneous immediate and delayed hypersensitivity to chlorhexidine digluconate. Contact Dermatitis 44: 59

39. Bae YJ, Park CS, Lee JK, Jeong E, Kim TB, et al. (2008) A case of anaphylaxis to chlorhexidine during digital rectal examination. J Korean Med Sci 23: 526 528 .

40. Yusof WZ, Khoo SP (1988) Mucosal sensitivity to chlorhexidine mouthwash Singapore Dent J 13: 39-40.

41. Sharma A, Chopra H (2009) Chlorhexidine urticaria: a rare occurrence with a common mouthwash. Indian J Dent Res 20: 377-379.

42. Goon AT, White IR, Rycroft RJ, McFadden JP (2004) Allergic contact dermatitis from chlorhexidine. Dermatitis 15: 45-47.

43. Ramselaar CG, Craenen A, Bijleveld RT (1992) Severe allergic reaction to an intraurethral preparation containing chlorhexidine. Br J Urol 70: 451-452. 
Citation: Calogiuri GF, Di Leo E, Trautmann A, Nettis E, Ferrannini A, et al. (2013) Chlorhexidine Hypersensitivity: A Critical and Updated Review. J Allergy Ther 4: 141. doi:10.4172/2155-6121.1000141

44. Mitchell DJ, Parker FC (1993) Anaphylaxis following urethral catheterisation Br J Urol 71: 613

45. Russ BR, Maddern PJ (1994) Anaphylactic reaction to chlorhexidine in urinary catheter lubricant. Anaesth Intensive Care 22: 611-612.

46. Yong D, Parker FC, Foran SM (1995) Severe allergic reactions and intraurethral chlorhexidine gluconate. Med J Aust 162: 257-258.

47. Jayathillake A, Mason DF, Broome K (2003) Allergy to chlorhexidine gluconate in urethral gel: report of four cases and review of the literature. Urology 61: 837

48. Knight BA, Puy R, Douglass J, O'Hehir RE, Thien F (2001) Chlorhexidine anaphylaxis: a case report and review of the literature. Intern Med J 31: 436437

49. Ebo DG, Bridts CH, Stevens WJ (2004) Anaphylaxis to an urethral lubricant: chlorhexidine as the "hidden" allergen. Acta Clin Belg 59: 358-360.

50. Parkes AW, Harper N, Herwadkar A, Pumphrey R (2009) Anaphylaxis to the chlorhexidine component of Instillagel: a case series. Br J Anaesth 102: 65-68.

51. Oda T, Hamasaki J, Kanda N, Mikami K (1997) Anaphylactic shock induced by an antiseptic-coated central venous [correction of nervous] catheter. Anesthesiology 87: 1242-1244.

52. Maki DG, Ringer M, Alvarado CJ (1991) Prospective randomised trial of povidone-iodine, alcohol, and chlorhexidine for prevention of infection associated with central venous and arterial catheters. Lancet 338: 339-343.

53. Terazawa E, Shimonaka H, Nagase K, Masue T, Dohi S (1998) Severe anaphylactic reaction due to a chlorhexidine-impregnated central venous catheter. Anesthesiology 89: 1296-1298.

54. Lockhart AS, Harle CC (2001) Anaphylactic reactions due to chlorhexidine allergy. Br J Anaesth 87: 940-941.

55. Pittaway A, Ford S (2002) Allergy to chlorhexidine-coated central venous catheters revisited. $\mathrm{Br} \mathrm{J}$ Anaesth 88: 304-305.

56. Khoo A, Oziemski P (2011) Chlorhexidine impregnated central venous catheter inducing an anaphylatic shock in the intensive care unit. Heart Lung Circ 20 $669-670$

57. Beatty P, Kumar N, Ronald A (2011) A complicated case of chlorhexidineassociated anaphylaxis. Anaesthesia 66: 60-61.

58. Jee R, Nel L, Gnanakumaran G, Williams A, Eren E (2009) Four cases of anaphylaxis to chlorhexidine impregnated central venous catheters: a case cluster or the tip of the iceberg? Br J Anaesth 103: 614-615.

59. Bilò MB, Cinti B, Chiarello M, Bonifazi F, Moneret-Vautrin DA (2005) Intraoperative anaphylaxis: verba volant, scripta manent! Eur Ann Allergy Clin Immunol 37: 339-340.

60. Nishioka K, Doi T, Katayama I (1984) Histamine release in contact urticaria Contact Dermatitis 11: 191

61. Layton GT, Stanworth DR, Amos HE (1989) The incidence of IgE and IgG antibodies to chlorhexidine. Clin Exp Allergy 19: 307-314

62. Pham NH, Weiner JM, Reisner GS, Baldo BA (2000) Anaphylaxis to chlorhexidine. Case report. Implication of immunoglobulin $\mathrm{E}$ antibodies and identification of an allergenic determinant. Clin Exp Allergy 30: 1001-1007.

63. Layton GT, Stanworth DR, Amos HE (1987) The specificity of murine polyclonal and monoclonal antibodies to the haptenic drug chlorhexidine induced by chlorine-generated chlorhexidine-protein conjugates. Clin Exp Immunol 69: 157-165.

64. Olivieri J, Eigenmann PA, Hauser C (1998) Severe anaphylaxis to a new disinfectant: polyhexanide, a chlorhexidine polymer. Schweiz Med Wochenschr 128: 1508-1511.

65. Ferrarini A, Baggi M, Flückiger R, Bianchetti MG (2006) Intraoperative anaphylaxis to a chlorhexdine polymer in childhood. Paediatr Anaesth 16: 705.
66. Kautz O, Schumann H, Degerbeck F, Venemalm L, Jakob T (2010) Severe anaphylaxis to the antiseptic polyhexanide. Allergy 65: 1068-1070.

67. Waclawski ER, McAlpine LG, Thomson NC (1989) Occupational asthma in nurses caused by chlorhexidine and alcohol aerosols. BMJ 298: 929-930.

68. Sato K, Kusaka Y, Suganuma N, Nagasawa S, Deguchi Y (2004) Occupational allergy in medical doctors. J Occup Health 46: 165-170.

69. Garvey LH, Roed-Petersen J, Husum B (2003) Is there a risk of sensitization and allergy to chlorhexidine in health care workers? Acta Anaesthesiol Scand 47: $720-724$

70. Garvey LH, Roed-Petersen J, Menné T, Husum B (2001) Danish Anaesthesia Allergy Centre - preliminary results. Acta Anaesthesiol Scand 45: 1204-1209.

71. Nagendran V, Wicking J, Ekbote A, Onyekwe T, Garvey LH (2009) IgEmediated chlorhexidine allergy: a new occupational hazard? Occup Med (Lond) 59: $270-272$

72. Liippo J, Kousa P, Lammintausta K (2011) The relevance of chlorhexidine contact allergy. Contact Dermatitis 64: 229-234.

73. McCoy LC, Wehler CJ, Rich SE, Garcia RI, Miller DR, et al. (2008) Adverse events associated with chlorhexidine use: results from the Department of Veterans Affairs Dental Diabetes Study. J Am Dent Assoc 139: 178-183.

74. Kahn CR, Schecther $Y$ (1991) Insulin, oral hypoglycemic agents and the pharmacology of endocrine pancreas. In: Goodman and Gilman's The Pharmacology Basis of Therapeutics (8th Edn) Pergamon Press New York: 1463-1495.

75. Florvaag E, Johansson SG (2009) The pholcodine story. Immunol Allergy Clin North Am 29: 419-427.

76. Garvey LH, Krøigaard M, Poulsen LK, Skov PS, Mosbech H, et al. (2007) lgEmediated allergy to chlorhexidine. J Allergy Clin Immunol 120: 409-415.

77. Nakonechna A, Dore P, Dixon T, Khan S, Deacock S, et al. (2012) Immediate hypersensitivity to chlorhexidine is increasingly recognised in the United Kingdom. Allergol Immunopathol (Madr) .

78. Ebo DG, Bridts CH, Stevens WJ (2006) IgE-mediated anaphylaxis from chlorhexidine: diagnostic possibilities. Contact Dermatitis 55: 301-302.

79. Noel J, Temple A, Laycock GJ (2012) A case report of anaphylaxis to chlorhexidine during urinary catheterisation. Ann R Coll Surg Engl 94: e159160 .

80. Sáenz de San Pedro B, Mayorga C, Torres MJ, Florido JF, Quiralte J, et al (2002) Boosted IgE response after anaphylaxis reaction to cefuroxime with cross-reactivity with cefotaxime. Ann Allergy Asthma Immunol 89: 101-103.

81. Calogiuri GF, Nettis E, Di Leo E, Vacca A, Ferrannini A, et al. (2012) Kounis Syndrome induced by intravenous administration of piperacillin/tazobactam: a case report. Int J Cardiol 155: e42-44.

82. Bommer J, Ritz E (1987) Ethylene oxide (ETO) as a major cause of anaphylactoid reactions in dialysis (a review). Artif Organs 11: 111-117.

83. Aalto-Korte K, Mäkinen-Kiljunen S (2006) Symptoms of immediate chlorhexidine hypersensitivity in patients with a positive prick test. Contact Dermatitis 55: 173-177.

84. De Queiroz M, Combet S, Bérard J, Pouyau A, Genest H, et al. (2009) Latex allergy in children: modalities and prevention. Paediatr Anaesth 19: 313-319.

85. Wittczak T, Dudek W, Walusiak-Skorupa J, Swierczynska-Machura D Palczynski C (2013) Chlorhexidine--still an underestimated allergic hazard for health care professionals. Occup Med (Lond) 63: 301-305.

86. Harper NJ, Dixon T, Dugué P, Edgar DM, Fay A, et al. (2009) Suspected anaphylactic reactions associated with anaesthesia. Anaesthesia 64: 199-211.
This article was originally published in a special issue, Drug Allergy handled by Editor. Dr. Helieh S Oz, University of Kentucky Medical Center, USA 